\title{
Filmdiskussionen als (Forschungs-)Methode in der Sozialen Arbeit
}

\author{
Wie Filme einen narrativ orientierten Zugang zu \\ Bewältigungsanforderungen im Alltag jugendlicher Geflüchteter \\ eröffnen
}

\author{
Heidrun Schulze • Tanja Grendel
}

Online publiziert: 11. Mai 2020

(C) Der/die Autor(en) 2020

Zusammenfassung Häufig ist es für Jugendliche mit Fluchterfahrung nicht leicht, eigene Ausgrenzungserfahrungen zu thematisieren, was u.a. auf Deutungsmuster zurückzuführen ist, die auf gesellschaftlichen Macht- und Herrschaftsverhältnissen beruhen. Diese Deutungsmuster weisen Geflüchteten - im Gegensatz zu bereits etablierten Mitgliedern der Gesellschaft - eine marginalisierte Position zu, schreiben sich in die alltägliche Lebenspraxis ein und erwarten von den Adressat*innen die Übernahme identitärer Zuweisungen. Für eine emanzipatorische Bildung stellt sich die Herausforderung, wie eine Verständigung über die individuellen Lebensinteressen geflüchteter Jugendlicher und die potenziell verhindernden gesellschaftlichen Lebensverhältnisse, aber auch die Bewältigungsformen der Jugendlichen zur Sprache kommen können, ohne in eine aufklärerische Rekolonisierung abzudriften. Ausgehend von einem rekonstruktiven Praxis-Forschungs-Projekt in der Sozialen Arbeit zeigt der vorliegende Beitrag auf, wie Filmdiskussionen einen Zugang zu Diskriminierungs- bzw. Rassismuserfahrungen von jugendlichen Geflüchteten eröffnen. Am Beispiel einer transkribierten Filmdiskussion wird dokumentiert, wie sich Merkmale der Methode der Filmdiskussion - mit dem Film als Stimulus, einer narrativ orientierten Gesprächsführung und dem Gruppensetting - auf die Thematisierbarkeit subjektiver und kollektiver Erfahrungen von Ausgrenzung auswirken und Bildungsprozesse mit emanzipatorischem Potenzial initiieren können.

Schlüsselwörter Jugendliche Geflüchtete · Filmdiskussionen · Diskriminierung · Praxis-Forschungsmethode $\cdot$ transformative Bildung

Prof. Dr. H. Schulze $(\bowtie) \cdot$ Prof. Dr. T. Grendel

Hochschule RheinMain, Kurt-Schumacher Ring 18, 65197 Wiesbaden, Deutschland

E-Mail: Heidrun.schulze@hs-rm.de

Prof. Dr. T. Grendel

E-Mail: Tanja.grendel@hs-rm.de 


\title{
Film-related discussions as a (research-)method in social work
}

How films offer narrative-oriented access to coping requirements in the everyday lifes of young refugees

\begin{abstract}
It is often difficult for adolescents with refugee experiences to discuss their own experiences of exclusion, which can be traced back to patterns of interpretation based on social power relations, among other things. In contrast to established members of society, these patterns of interpretation marginalize refugees, inscribe themselves in their everyday life experiences, and expect the addressees to adopt assigned identities. The challenge for emancipatory education is how to address an understanding of the individual life interests of refugees and social living conditions that could potentially impede them from pursuing these interests as well as the coping mechanisms of the adolescents without drifting off into a discourse of illuminating recolonization. Starting from a reconstructive practice-research project in the field of social work, this article shows how film-related discussions open up access to experiences of discrimination and racism among adolescent refugees. Using the example of a transcribed film-related discussion, the article documents how the characteristics of the method of film-related discussion - with the film as a stimulus, a narrative oriented conversation, and a group setting - can affect the way in which subjective and collective experiences of exclusion can be addressed and initiate educational processes with emancipatory potential.
\end{abstract}

Keywords Young refugees · Film discussion · Discrimination · Practice-research project $\cdot$ Transformative education

Mit dem vorliegenden Beitrag zeigen wir ausgehend von dem Praxis- wie Forschungsprojekt „Lernort Kino - Filmbildung mit Geflüchteten Jugendlichen“ ${ }^{\text {" }}$ auf, wie Filmdiskussionen einen subjektorientierten Zugang zu den Erfahrungen in der alltäglichen Lebenspraxis geflüchteter Jugendlichen mit Blick auf gesellschaftlichpolitische Verhältnisse eröffnen (können).

In der Kombination von Praxismethode (,Filmdiskussion“) und Forschungsmethode (,Gruppendiskussion“) beabsichtigten wir, für die Jugendlichen einen Denkund Artikulationsraum für den selbstgesteuerten Austausch von (Be-)Wertungen und Interpretationen der Filmthemen herzustellen und eine Brücke zur eigenen erlebten Realität zu bauen. Bei der Rahmung durch Film, Setting und Gesprächshaltung ging es uns insbesondere darum, die Jugendlichen nicht als zu „Belehrende“ zu positionieren. Ansinnen der Praxis- und der rekonstruktiven Forschungsmethode war es, zunächst einen Verstehenszugang zu den Perspektiven der Jugendlichen und ihren Lebensdeutungen und den zugrunde liegenden Bedingungen zu schaffen.

\footnotetext{
1 Das Projekt wurde 2016 bis 2017 in Kooperation von MIK Netzwerkarbeit im Berufsschulzentrum Wiesbaden e. V., Friedrich-Wilhelm-Murnau-Stiftung und der Freiwilligen Selbstkontrolle der Filmwirtschaft mit der Hochschule RheinMain durchgeführt. Das in diesem Beitrag diskutierte Forschungsprojekt an der Hochschule RheinMain Wiesbaden wurde durch das Kulturamt der Stadt Wiesbaden gefördert.
} 
Zunächst skizzieren wir den Umgang mit Diskrimierung(serfahrungen) in der Sozialen Arbeit (1) und den Forschungsstand zum Einsatz von Filmen bzw. Filmdiskussionen in der Sozialen Arbeit (2) und stellen nachfolgend Vorgehen sowie Ergebnisse der Analyse unserer Filmdiskussion mit Bezügen zur qualitativen Forschung und rekonstruktiven Zugängen vor (3). Das abschließende Fazit reflektiert die Forschungsmethode der Filmdiskussion im Kontext der Sozialen Arbeit mit jugendlichen Geflüchteten. Herausgearbeitet werden insbesondere deren narrative Selbstverständigungsprozesse hinsichtlich subjektiver Probleme in der Bewältigung gesellschaftlicher Lebensverhältnisse, welche dem Anspruch einer emanzipatorischen Praxisforschung und Bildungsarbeit im Sinne der Reflexion eigenen Denkens und Handelns mit dem Ziel der Veränderung restriktiver Lebensbedingungen folgen (4).

\section{Sprechen mit geflüchteten Jugendlichen als Zugang der Sozialen Arbeit zu Diskrimierung(serfahrungen)}

Im Kontext von Flucht, bei Bezugnahme auf geflüchtete Menschen, herrscht vielerorts - auch in der Pädagogik - eine Praxis der ,,bedürfniszentrierten Rede“ (Fraser 1994, S. 250ff.) als Modus hegemonialer Interpretationen Geflüchteter, in der Letztere mittels professioneller Narrative bezüglich Bildung und Sprache als defizitäre Subjekte konstruiert werden. Eine solche - in Praxis und Wissenschaft durchaus übliche - homogenisierende Interpretation der Bedarfe geflüchteter junger Menschen durch Expert*innen steht allen Selbstbestimmungs- und Partizipationsansprüchen entgegen. Wie bei vielen anderen Menschen, insbesondere aber jenen mit geringer sozialer, politischer und wirtschaftlicher Macht, wird - entgegen der Forderung von Bundesjugendkuratorium (BJK 2009), UN-Kinderrechtskommission (UN 1989) und International Organization of Migration (IOM 2013) - in öffentlichen Diskursen eher für oder über geflüchtete junge Menschen gesprochen, aber selten mit ihnen als Akteur*innen ihres eigenen Migrationsprojekts. Das professionelle „Narrativ“ über sie ist in der Regel entweder negativ, verallgemeinernd oder eingeschränkt; ihre Geschichten sind Defizitgeschichten, von anderen für sie und über sie ,geschrieben“ (,written“) (Rappaport 1995, S. 796). Die selbstbestimmte Nutzung von Artikulationsräumen, in denen geflüchtete junge Menschen ihre individuellen und kollektiven Stimmen und Interessen äußern können, auf ein anerkennendes „,Gehörtwerden“ (Dörr 2008) treffen und vor allem als einflussnehmende Akteur*innen im politischen Raum gesehen werden, wird ihnen in pädagogischen Arrangements nur selten - im öffentlich-politischen Raum bisher gar nicht (Liebel 2015) - eröffnet. Oder es wird so gestaltet, dass beim Sprechen mit ihnen schon vorab das Thema „Migrationshintergrund“ in den Mittelpunkt gestellt und von Pädagog*innen durch die Problematisierung der Lebenssituation mittels homogenisierender, national konstruierter Identitätskategorien von einem Außenstandpunkt fokussiert wird, mithin also soziale Ungleichheit durch Deutungshoheit wieder reproduziert wird.

Sprechen ist nicht ,unschuldig ' oder , unbedarft ", sondern Sprache und Macht sind in pädagogischen Settings untrennbar miteinander verknüpft, mit Sprache können wir gesellschaftliche Verhältnisse stabilisieren, aber auch verändern, wie es Hans- 
Rüdiger Müller (2011) unter Bezug auf Judith Butlers (2006) Konzept der Performativität formuliert: „Sprache ist das konstitutive Element sowohl für Veränderung gesellschaftlicher Verhältnisse als auch die (temporäre) Stabilisierung dieser Verhältnisse und wird als aktives Mittel verstanden, Transformationen und Stabilisierungen des Sozialen zu vollziehen. Ihr wohnt ein genuin emanzipatorisches und kritisches Potential inne“ (Müller 2011, o. S.; auch Butler 2006, S. 249 ff.). Wie wir über die Dinge sprechen, ,macht' die Dinge (auch) zu dem, was sie ,sind ‘. Zu Fragen ist: Wie wird etwas aus den Augen bestimmter Akteur*innen im Rückgriff auf selbstverständliche Diskurse und Standpunkte sagbar gemacht? Und: Was wird wie sagbar gemacht? Was wird wie als sagbar ausgeschlossen? Denn: wenn z. B. Rassismus als Phänomen nicht benannt werden darf, ist die Benennung erlebter Alltagsrassismen nur schwer möglich und wird aus der gesellschaftlichen Realität ausgeschlossen. Das Sprechen über Rassismus - und damit auch dessen Identifizierung - zu erschweren, ist nicht nur funktional für Rassismus, sondern ein Teil des Rassismus selbst (Kilomba 2009). Ähnlich den Gelingensbedingungen der Subordinierung von Frauen durch den gesellschaftlichen Diskurs zu gewaltvollem Sexismus (Langton 2007) wird Geflüchteten das symbolische Kapital eines legitim(iert)en Sprechens über Rassismus entzogen, was ein Verstummen zur Folge haben kann. Aber das Sprechen kann auch zum Verstummen führen, wenn die Thematisierung erlebter oder beobachteter rassistischer Diskriminierung in pädagogischen wie therapie(nahen) Kontexten fehlt (Mecheril 2010) oder - wie Sprachanalysen zeigen - beim Sprechen über Rassismus bestehende und erlebte Hierarchien fortgeschrieben werden (Kupfer 2018; Schulze et al. 2016).

Für Jugendliche mit Fluchterfahrung ist es oftmals nicht leicht, eigene Rassismus- bzw. Diskriminierungserfahrungen zu thematisieren, da sie sich aufgrund der Erwartungen der Aufnahmegesellschaft veranlasst fühlen, Dankbarkeit für die ,Aufnahme' zeigen zu müssen. Hinzu kommt, dass diese jungen Menschen - sozial und politisch - vorwiegend entsprechend den Kategorien ,Flüchtling ' und/oder ,Ausländer*in' wahrgenommen werden (Grendel 2018). Diese Kategorien sind mit Zugehörigkeitsdiskursen verknüpft, die häufig eine Vorrechtsstellung von Angehörigen der sogenannten ,Mehrheitsgesellschaft' zum Ausdruck bringen.

Norbert Elias und John L. Scotson (2006) bezeichnen dieses Phänomen als „Etabliertenvorrechte“, die auf der Vorstellung beruhen, dass neu hinzukommenden Menschen in einer Gesellschaft weniger Rechte zustehen als jenen, die bereits etabliert sind (vgl. auch Heitmeyer 2002, S. 20). Die Spezifik des Beziehungstyps „Etablierte-Außenseiter" basiert auf einer Komplementarität, die gekennzeichnet ist durch die Praxis der Abwertung ,Zugezogene*r" zur Aufrechterhaltung einer sozialen Überlegenheit der etablierten Gruppe. Mitunter werden in diesem Kontext Kultur und Staatsbürgerschaft als Medium der Zuweisung gesellschaftlicher Positionen und zur Behauptung einer gesellschaftlichen Position eingesetzt und für Abwertungspraxen genutzt. Menschen mit Fluchtgeschichte sind entsprechend mit dem Ausschluss gleichberechtigter Teilhabe konfrontiert, die auf gesellschaftlichen Vorstellungen von Vorrechten beruht. Ihre Anspruchsaktivitäten zu gleichberechtiger Teilhabe werden von Mehrheitsangehörigen als anmaßend und unzulässig bewertet.

Verschiedene Konzepte und Analysen zeigen, dass Menschen mit Migrationsgeschichte diese Vorstellungen häufig internalisieren, sich in spezifischer Weise mit 
dem (kultur)rassifizierenden Homogensierungsdiskurs in Beziehung setzen und in der Folge sich selbst als anomisch, ,schlecht' wahrnehmen (u.a. Rommelspacher 2006, 1995). „Das ist, glaube ich, das ist unsere Schuld, das ist Schuld von Ausländern“, sagte auch ein jugendlicher Teilnehmer in unserem Projekt, „manche Ausländer sind sehr schlechte [...], dann wir müssen jetzt zeigen, dass die Ausländer sind auch nicht nur schlechte, wir müssen was gute machen für die Deutschland“ (Jugendlicher 4, Z. 647-651). Damit unterstreicht der Jugendliche, dass er die Zuschreibung der Nichteinhaltung aufgestellter Normen internalisiert hat, die die Mitglieder der Etabliertengruppen ihm - dem „Dazugekommenen“ - gegenüber zum Ausdruck bringen, um ihre eigene Macht zu stabilisieren (Elias und Scotson 2006, S. 18). Es ist denkbar, dass er die von der Mehrheitsgesellschaft aufgestellten Werte akzeptiert und deren Perspektive verinnerlicht hat, wonach die Mitglieder seiner religiös-nationalen Herkunfts- und geschlechtlichen Gemeinschaft - stereotypisierend und alienisierend als Männer mit ,arabischem Aussehen ' - minderwertig sind.

Internalisierung bezweckt nach N. Elias und J. L. Scotson (2006) eine Hoffnung, an der als besser erachteten Gruppe - in diesem Beispiel den ,nicht schlechten * Einheimischen, den ,Deutschen', deren Konstruktion mit der hegemonialen Selbstkonstruktion ,schlechte Ausländer' in Relation steht - zu partizipieren, um sich aus dem mit ,Schuld' behafteten und entwürdigten Minderheitenstatus zu emanzipieren. Der normative Diskurs der Mehrheitsgesellschaft wird damit keineswegs verlassen, sondern laut J. Butler (2001) in einer Art ,Selbstkolonisierung“ (Butler 2001, S. 99) übernommen: „Angerufen durch einen verletzenden Namen erhalte ich ein soziales Dasein, und weil ich eine gewisse unumgängliche Verhaftung mit meinem Dasein habe [...], begrüße ich schließlich die mich verletzenden Bedingungen, denn sie konstituieren mich sozial“ (Butler 2001, S. 99). Dadurch „erscheint die Selbstbeziehung des Subjekts, sein reflexives Selbstverhältnis, als Verlängerung der Macht, die von außen auf das Individuum einwirkt“ (Kämpf 2006, S. 352). Mit der Übernahme der Überzeugung, dass die anderen besser seien, ist die Hoffnung auf Teilhabe an der idealisierten und ausgrenzenden Gruppe verbunden. Anknüpfend an N. Elias und J. L. Scotson (2006) könnte es sich hierbei um einen Versuch der persönlichen Destigmatisierung durch Übertragung der Stigmatisierungskategorien auf die eigene Herkunftsgruppe und durch individuelle Distanzierung von kollektiven, durch die Mehrheit begründeten Konstruktionen einer Gruppe, handeln. Mögliche Folgen dieser Internalisierung sind ein starkes Bemühen um Assimilation (vgl. Weiß 2001, S. 104), die Vernachlässigung eigener Ansprüche, Wünsche und Ziele (vgl. Grendel 2018; Horvath 2017; Weiß 2013; Niedrig und Seukwa 2010, S. 191; Weiß 2001) sowie die - von Mehrheitsangehörigen erwartete - Verleugnung von Rassismus als Teil von Alltagsrassismus (Melter 2006), der das Erleben rassistischer Praxis bagatellisiert und z. T. psychologisierend individualisiert (Schulze 2009). Vor diesem Hintergrund wird zum einen die Thematisierung von Rassismus- bzw. Diskriminierungserfahrungen erschwert, da sie teilweise im Einklang mit gesellschaftlichen Vorstellungen stehen (Bourdieu 1982/1990). Zum anderen kann dies zu einer Beschämung führen aufgrund der sozialpolitischen und verletzbaren Positionen potenziellen „Betroffenseins“ von Rassismus, denn bereits das Aussprechen trifft auf Beschwichtigungen, Bagatellisierungen (u. a. Schramkowski 2007; Rosenthal 2002, S. 151) und wieder Stigmatisierungen (Schulze und Witek 2014; Neckel 1991). 
Für die Soziale Arbeit ist ein Zugang zu den Alltagserfahrungen sowie etwaigen Diskriminierungserfahrungen jugendlicher Geflüchteter unerlässlich, dies leitet sich aus dem Auftrag der Profession ab, der in der Arbeit mit Individuen und an ausgrenzenden Strukturen besteht (vgl. Schramkowski und Ihring 2018). Ziel ist die Förderung sowohl personaler Selbstbestimmung als auch sozialer Integration. Professionelle bewegen sich folglich in einem Spannungsfeld zwischen der koproduktiven Ermöglichung individueller Ziele und Vorhaben einerseits und der Aufdeckung und Einbindung in soziale Strukturen andererseits (vgl. Ziegler 2015, S. 453) - gemeinsam mit den Adressat*innen. Dies setzt eine kritische Reflexion gesellschaftlicher Strukturen im Sinne potenzieller Begrenzungen von Selbstbestimmung und Integration zusammen mit den Adressat*innen voraus, in unserem Beispiel die Vermeidung von Individualisierung und paternalistischer Pädagogik. Wollen wir die Perspektive vom Standpunkt der geflüchteten Jugendlichen erkunden, ihr Verhältnis zu ihren strukturellen, diskursiven und interaktionellen Erfahrungen und Bewältigungen angesichts von Rassismus- sowie Diskriminierungspraxen und die daraus folgenden restriktiven Bedingungen der sozialen Teilhabe für alle Beteiligten - also auch für die Moderator*innen der Gruppendiskussion - in die Wahrnehmung bringen, so gilt es, einen Verständigungsraum im Sinne eines kritischen transformatorischen Bildungsraums (vgl. Sting 2018) zu etablieren. Der Blick dabei ist auf den potentiellen Nutzen der geflüchteten Jugendlichen zu richten und dahingehend zu hinterfragen. Darunter verstehen wir die individuelle Bewusstmachung und Veränderung ausgrenzender Strukturen für die Jugendlichen selbst wie auch für die Weiterentwicklung der Sozialer Arbeit im Fluchtkontext in Sinne einer subjektorientierten und alltagskritischen Adressat*innenorientierung.

\section{Filmdiskussionen als Zugang zu Adressat*innen in der Sozialen Arbeit}

Mit Filmen wird in der Sozialen Arbeit in unterschiedlichen Kontexten und mit unterschiedlichen Zielsetzungen gearbeitet. Das vorliegende Praxis-Forschungs-Projekt fokussiert auf das Potenzial des methodischen Einsatzes von Filmen in der Arbeit mit jugendlichen Geflüchteten und darüber hinaus auf die empirische Rekonstruktion des dialogisch orientierten Interaktionsprozesses der anschließenden Gruppendiskussion. Ausgehend von einer Systematisierung unterschiedlicher pädagogischer Einsatzmöglichkeiten von Filmen wird auf die Filmdiskussion eingegangen bzw. auf die den jugendlichen Geflüchteten hierdurch eröffnete Möglichkeit, sich zum Film und zu den für sie darin relevanten Themen bzw. Figuren im Gruppensetting wie auch gesellschaftlich zu positionieren und diese mit ihren eigenen alltäglichen Erfahrungen und Bewältigungsanforderungen in Beziehung zu setzen.

Einer der Ansätze orientiert sich an der „Kultivierungsthese“ (Bonfadelli 1983; Gerbner und Gross 1976), die davon ausgeht, dass die Mediennutzung eine bestimmte Wahrnehmung der Welt prägt. Dieser Perspektive lassen sich im Migrations- bzw. Geflüchtetenkontext u.a. Ansätze zuordnen, die Filme einsetzen, um Einblicke in den Alltag sowie gesellschaftliche Normen und Werte der Aufnahmegesellschaft zu 
vermitteln (vgl. bpb 2016), im Sinne der Aufklärung und Belehrung über Werte und zur Anpassung an diese in der Dominanzgesellschaft.

Filme können des Weiteren einen Einfluss auf eine kritische Bewusstseinsbildung über gesellschaftliche Verhältnisse und Welt-Selbst-Erleben ausüben und verändernd wirken. Dies beinhaltet die Wahrnehmung und Reflexion der eigenen Position innerhalb gesellschaftlicher Machtstrukturen und birgt das Potenzial, die Reproduktion sozialer Ungleichheit zu beeinflussen (vgl. Süss et al. 2013, S. 47 ff.). Ansätze, die sich an dieser Ausrichtung orientieren, folgen nicht zuletzt dem Anspruch politischer Bildung und intendieren die Emanzipation der Rezipient*innen (vgl. Walber 2011).

Darüber hinaus werden in der Fachliteratur Filme (sowie weitere Medien) im Zusammenhang mit sozialer Selbstentwicklung gesehen: Filmfiguren werden als Identifikationsressourcen im gesellschaftlich vermittelten Orientierungsprozess über sich selbst verstanden, die die Spiegelung eigener biografischer Erfahrungen ermöglichen, aber auch als Orientierungshilfen dienen können (vgl. Süss 2006, S. 337). Vor diesem Hintergrund werden Filme z. B. eingesetzt, um Anregungen für die Ausgestaltung der eigenen Geschlechterrolle zu geben (vgl. u. a. Luca und Decke-Cornill 2010; Bilden 2007; Luca 2003; Barthelmes und Sander 2001, S. 147-158). Durch die Identifikation mit den Filmfiguren können die Jugendlichen stellvertretende Erfahrungen machen und Ähnlichkeiten zwischen der eigenen Situation bzw. eigenen Erfahrungen und denen der Filmfiguren reflektieren (vgl. Hébert und Neumeister 2002, 2001). Ausgehend von den Annahmen der Theorie sozialen Vergleichs ist ferner davon auszugehen, dass Filmfiguren einen Einfluss auf die Bewertung der eigenen Person bzw. von sozialen Phänomenen und Situation ausüben können (vgl. Festinger 1954).

Im pädagogischen Kontext spielt zudem eine Rolle, dass nicht nur der Film selbst, sondern auch die anschließende Filmdiskussion Impulse setzen kann, um das eigene Verhältnis in Bezug auf erweiterte Deutungsmöglichkeiten und erweiterte Handlungsmöglichkeiten gegenüber restriktiven Bedingungen - in unserem Beispiel: einer möglichen hierarchischen Ausgrenzungs- und Diskriminierungspraxis $-\mathrm{zu}$ reflektieren. Film und Filmdiskussion können den Jugendlichen auch einen Gegendiskurs anbieten, der ihnen die Möglichkeit der Selbstvergewisserung über Subjektives und über ihre gesellschaftlichen Verhältnisse eröffnet, bis hin zu einer Wiedererlangung von Handlungswirksamkeit und Würde.

Ein Ziel der Arbeit mit Filmen als Methode in der Sozialen Arbeit besteht also darin, dass die Teilnehmenden sich selbst bewusst werden über die gesellschaftlichmachtpolitischen Verhältnisse, in die ihre subjektiven Erfahrungen eingebettet sind, aber auch die Möglichkeit des Einflusses auf deren Veränderbarkeit, mit dem Ziel der Verwirklichung ihrer Lebensinteressen. Die gemeinsame Filmerfahrung kann einen Raum für das gemeinsame Sprechen und die Verallgemeinerung eines scheinbar individuellen Leids an den bestehenden Verhältnissen anbieten. Die Frage ,Was sind die Bedingungen, unter denen die Probleme entstehen?“ (Osterkamp 2018, S. 93) sei erst angebracht auf eine subjektive Äußerung wie „Das kenne ich, das habe ich auch, was können wir dagegen machen?“, so Ute Osterkamp (2018), da in dieser Äußerung bereits ,eine Art von Verallgemeinerung“ enthalten sei. Angeregt durch Situationen im Film und das reziproke Sich-in-Verhältnis-Setzen zu 
konkreten Situationen des eigenen Lebens können eine Problembewusstheit und die Frage nach ähnlichen eigenen Lebensbedingungen - und damit auch Handlungsimpulse - entstehen, auch wenn die Fluchtgeschichten sich voneinander unterscheiden. Über die Filmfiguren wie auch die weiteren Gesprächsteilnehmer*innen kann sich den Teilnehmer*innen - in gewisser Weise doppelt legitimiert - die Erfahrung vermitteln, nicht ,alleine“ zu sein. In der Folge können Ausgrenzungserfahrungen als strukturell statt individuell bedingt reflektiert werden. Die Kollektiverfahrung birgt darüber hinaus das Potenzial, dass die Jugendlichen sich als machtvoll erleben und eine gemeinsame Stimme ausbilden. So bietet das Gruppensetting die Möglichkeit, bei den Jugendlichen das Bewusstsein ihrer kollektiven Situation zu wecken, mit dem Ziel einer erweiterten Handlungsfähigkeit (Litau et al. 2016; Holzkamp 1985, S. 375) und der Fähigkeit, in ihr eigenes Leben - als eine strukturell gegebene und subjektiv erfahrene Lebenssituation - gestaltend eingreifen zu können. Die Bestärkung durch die anderen Jugendlichen, die zumindest die kollektive Erfahrung der sozialen Positionierung im Ankunftsland teilen, kann persönliche und kollektive (verallgemeinerte) Handlungsfähigkeit verstärken.

Neben der Identifikation mit Filmfiguren treten subjektive Deutungen und Relevanzen bezüglich des Films zutage, die oftmals mit biografischen Erfahrungen und deren Verarbeitung verknüpft sind. In der Gesprächssituation werden diese Deutungen aufgenommen und der Gruppe als Deutungsangebot unterbreitet. Die Gruppe als eine sich möglichst selbst stimulierende Auseinandersetzungsarena - ermöglicht den Teilnehmer*innen, sich selbst innerhalb eines breiteren Meinungsspektrums der Gruppe zu positionieren und zu relativieren, wodurch Prozesse der Vergewisserung über sich und die Welt angestoßen werden.

Dabei obliegt es den Jugendlichen selbst, über persönliche Erfahrungen zu sprechen oder nicht. Die Filmfiguren können als Identifikationsressourcen im Prozess der Identitätsentwicklung fungieren und neue Einsichten eröffnen, u. a. infolge der Perspektivenübernahme. Das Gruppensetting der Filmdiskussion ermöglicht zudem eine Selbstverortung innerhalb der Gruppe und ein Austausch verbindender Kollektiverfahrungen aufgrund konjunktiver Erfahrungsräume, die (politische) Bildungsprozesse stimulieren können.

\section{Projekt „Lernort Kino“: Filmdiskussion als Zugang zu Bewältigungsanforderungen im Alltag jugendlicher Geflüchteter}

Das Projekt „Lernort Kino - Filmbildung mit geflüchteten Jugendlichen“ erstreckte sich über ein Jahr und adressierte Jugendliche in InteA-Klassen einer Wiesbadener Schule. Einmal im Monat wurden von der Friedrich-Wilhelm-Murnau-Stiftung und der Freiwilligen Selbstkontrolle der Filmwirtschaft im Kino Filme gezeigt, die von ca. 80 geflüchteten Jugendlichen und ihren Lehrer*innen besucht wurden. Zu ausgewählten Terminen boten wir Filmdiskussionen an, die wir im Rahmen unseres Forschungsprojektes im Anschluss in einem separaten Raum des Kinos durchführten. Der vorliegende Artikel fokussiert auf eine der Gruppendiskussionen, die im Anschluss an den vom Filmregisseur Theodore Melfi produzierten Film „Hidden Figures“ stattfand. Filmsujet ist die Geschichte der Emanzipation dreier afroameri- 
kanischer Mathematikerinnen, die in den 1960er-Jahren bei der NASA arbeiteten. Der Film zeigt insbesondere deren Diskriminierungserfahrungen aufgrund von Hautfarbe und Geschlecht und beeinflusste auf diese Weise den inhaltlichen Rahmen der Diskussion. Ausgewählt wurde der Film u.a. aufgrund des von den Jugendlichen formulierten Wunsches, aktuelle Kinofilme zu sehen und keine weiteren coming-ofage-Filme, die aus ihrer Sicht nicht ,ihre“ Themen aufgreifen.

Die an der Filmdiskussion beteiligte gemischtgeschlechtliche Gruppe war zufällig zusammengesetzt aus Jugendlichen, die an dem Projekt „Lernort Kino“ teilnahmen und sich aus Interesse an der Gruppendiskussion im Anschluss an die gemeinsame Filmvorführung beteiligt haben. Insgesamt nahmen acht Jugendliche von 80 Teilnehmer*innen im Alter von 16 bis 18 Jahren teil; sie kamen aus unterschiedlichen Herkunftsländern, darunter Afghanistan, Syrien und Äthiopien, und verfügten entsprechend über unterschiedliche Aufenthaltstitel.

Unsere Gruppendiskussion wurde per Audiogerät aufgezeichnet und vollständig transkribiert. Anschließend wurde sie zunächst sequenziell nach Themenfolge, Argumentationsaufbau (Bohnsack 2007) und -strukturbildung des sich herausbildenden kollektiven Orientierungsrahmens, anschließend im Wechsel feinanalytisch auf Basis induktiver Kategorienbildung und themenzentrierend mittels deduktiver Kategorisierung des Datenmaterials analysiert, und schließlich wurde eine Prozessanalyse der Diskursorganisation durchgeführt. Die Auswertung erfolgte neben dem Forschungsteam durch eine gemischtgeschlechtliche Gruppe von Studierenden mit verschiedenen Migrationsgeschichten. Hintergrund war die Intention, die Interpretationsvielfalt durch unterschiedliche generationelle und transnationale Perspektiven auszuweiten, auch um Möglichkeiten einer Dezentrierung unserer Deutungshoheit als Repräsentantinnen der „Dominanzkultur“ (Rommelspacher 1995) zu eröffnen. Die hier angewandte Methode der Filmdiskussion zeichnet sich durch drei Merkmale aus: einen Film als Stimulus, das Gruppensetting und eine narrativ orientierte Gesprächsführung. Nachfolgend werden diese Zugänge theoretisch und empirisch diskutiert.

\subsection{Stimulus Film: Impulse für ein Sich-in-Beziehung-Setzen zu anderen und zu sich selbst}

Stimuli werden auch im Forschungskontext häufiger als Anregung einer Gruppendiskussion eingesetzt (vgl. Lamnek 2005). Im hier diskutierten Projekt wurde der Film als Stimulus und Input für eine anschließende Diskussion angewandt, da das Medium Film Metaphern anbietet, die von den Rezipient*innen sowohl abstrakt als auch persönlich bzw. erfahrungsbasiert aufgenommen werden können (Bierman et al. 2003). Daher wird die Methode der Filmdiskussion als ,group cinematherapy“ (Bierman et al. 2003) auch in therapeutischen Settings angewendet. Filmfiguren eignen sich zudem als Gegenstand sozialer Vergleichsprozesse, haben damit Einfluss auf die Selbstwahrnehmung und können alternative Handlungsoptionen aufzeigen oder eine analytische Perspektive auf Erfahrungen und deren strukturelle Lebensbedingungen eröffnen.

Die Analyse der Gruppendiskussion belegt, dass der Film als Stimulus eine gemeinsame Erfahrung der Jugendlichen begründet. Zunächst leitet sich hieraus ein 
gemeinsamer Gesprächsgegenstand ab. Der Film legt bestimmte Themen nahe. Im konkreten Fall nannten die Jugendlichen Rassismus (aufgrund von Hautfarbe und Geschlecht), Frauenrechte sowie Begabung/Können. Im Verlauf der Gruppendiskussion kristallisierten sich Rassismus bzw. Diskriminierungserfahrungen jedoch als Schwerpunktthema heraus. Obgleich der Film als Stimulus eine bestimmte thematische Engführung mit sich bringen kann, wählen die Jugendlichen letztlich vor dem Hintergrund ihrer subjektiven Relevanzen aus, welche der Themen sie aufgreifen möchten und welche nicht.

Wie bereits herausgearbeitet, eröffnet der Stimulus Film den Jugendlichen die Möglichkeit, auf abstrakte Themen des Films wie auch die Filmfiguren oder eigene Erfahrungen Bezug zu nehmen. Bei den konkreten Bezügen zeigte sich, dass die Filmfiguren vornehmlich im Vergleich zur eigenen Person betrachtet werden, im vorliegenden Fall wurden v.a. Ähnlichkeiten hervorgehoben, z. B. in Bezug auf das eigene Leistungsvermögen (Jugendliche 2, Z. 101-107), aber auch auf eigene Diskriminierungserfahrungen: „Ich habe eine selbe Geschichte, weil ich komme aus Afghanistan, und ich hab' im Iran [...] gewohnen mit meine Familie, und dann die iranischen Leute hat viele diskriminiert genauso" (Jugendlicher 3, Z. 126-131). Die Filmfiguren dienen als eine Art Folie, vor deren Hintergrund Ähnlichkeit und Unähnlichkeit zur eigenen Person identifiziert werden.

Des Weiteren zeigt die Analyse, dass das Hineinversetzen in die Perspektive der Filmfiguren auch die Reflexion eigener biografischer Deutungsmuster zur Folge hat. In Erinnerung an eine Filmszene, in der eine der Hauptfiguren für eine sozialklassierte „Putzfrau“ gehalten wird, sagte ein Diskussionsteilnehmer: ,Wenn ich [...] eine Mann oder eine Frau sehen, und dann zum ersten Mal ich sehe nicht [...] diese [...] nur, nur Kleidung oder nur ihr Styling“ (Jugendlicher 3, Z. 153-157). Diese Form der Vorverurteilung bzw. Bewertung von Menschen kommentiert er abschließend mit den Worten: „Das große Problem ist in ganze Welt“ (Jugendlicher 3, Z. 173). Die filmische Darstellung initiiert auf diese Weise (auch) Prozesse von kritischer Selbstreflexion und transformativer Bildung. Eine andere Jugendliche resümierte: „Wenn ich kam zu Deutschland, ich war ein bisschen Rassist über die afghanischen Leute“ (Jugendliche 2, Z. 847-848). Der Film ermöglicht offenbar den Zuschauenden das Einnehmen unterschiedlicher Perspektiven und öffnet einen analytischen Blick aus einer gewissen Distanz, die dazu beiträgt, dass - wie in diesem Fall die eigene Betroffenheit durch Stigmatisierung als sog. „Andere“ wie selbst erfahrene Ausgrenzungsmechanismen je nach Selbst- und Fremdpositionierung reflektiert werden. Festzuhalten bleibt, dass der Film als Basis der Diskussion einen wichtigen gemeinsamen Ausgangspunkt herstellte. Die Figuren wie auch die geschilderten Situationen bildeten Referenzpunkte der Diskussion.

\subsection{Gruppensetting: diskursiver Bildungsraum und Ermöglichung von Selbstvergewisserung}

Ein zentrales Potenzial des Gruppensettings ist darin zu sehen, dass die Teilnehmenden zugleich Zuschauer*innen und Sprecher*innen sind: Eigene Sichtweisen werden innerhalb des Gruppenkontextes wahrgenommen und bewertet und führen zur Selbstreflexion und Veränderung von Sichtweisen; gemeinsame Erfahrungen (in 
unserem Fall z. B. soziale Wahrnehmung im Aufnahmeland) werden hervorgehoben und tragen zur Herausbildung eines Gruppengefühls bei.

Methodisch eröffnen Gruppendiskussionen einen Einblick in die Konstruktionen sozialer Wirklichkeit, die in gemeinsamen Erzählungen hergestellt werden (vgl. Flick 2009, S. 248). Im Unterschied zu Einzelinterviews bedingt die Gruppendynamik, dass mitunter auch - z. B. infolge sozialer Erwünschtheit - „tabuisierte Anteile“ (Flick 2009, S. 248) von Meinungen und Einstellungen zum Tragen kommen. Zudem eröffnet sich die Möglichkeit, die Validierung von in der Gruppe nicht geteilten Ansichten und die entsprechenden Argumentationsstrukturen in die Analyse einzubeziehen (vgl. Flick 2009, S. 251). Auf diese Weise kann eruiert werden, mit welchen Argumenten es zur Meinungsbildung in der Gruppe kommt und welcher kollektive Orientierungsrahmen sich abbildet. Die Effekte der Methode leiten sich insbesondere aus der Gruppenkonstellation ab. Neben individuellen Anknüpfungspunkten bietet das gemeinsame Sprechen über Filme Möglichkeiten individueller Selbstvergewisserung im Sinne einer narrativen Identitätsarbeit (vgl. Lucius-Hoene und Deppermann 2002).

Ausgangspunkt der Gruppendiskussion sollte die Hinwendung zur subjektiven Erfahrung und zu deren Bedingungen im alltäglichen Leben der Teilnehmenden sein, als ,Schnittstelle objektiver Strukturen und subjektiver Verständnis- und Bewältigungsmuster" (Thiersch 1992, S. 47). Damit ist der gewählte narrative und hermeneutische Zugang in die von Hans Thiersch seit Ende der 1970er-Jahre entwickelte Metatheorie einer alltagskritischen Sozialpädagogik eingebettet. Auf der Grundlage der sog. „hermeneutisch-pragmatischen Erziehungswissenschaft" (Thiersch 1978, S. 48) werden Alltagshandeln und Selbstdeutungen hinsichtlich der konkreten Lebensbedingungen beleuchtet. Im Sinne einer kritisch-subjektorientierten und emanzipatorischen Alltagstheorie und einer daraus begründeten Praxisforschung ging es uns darum, die Teilnehmenden dazu anzuregen, ihre Selbstdeutungen und Bewältigungsformen ins Verhältnis zu konkreten gesellschaftlichen Bedingungen zu setzen. Um dies zu realisieren, intendierten wir einen möglichst offenen, durch die Wirkung des Films angeregten Artikulationsraum zu schaffen, in dem die Anforderungen und Zumutungen des Alltags als aussprechbar erlebt werden, insbesondere die Begründungszusammenhänge im Umgang mit Ausgrenzungserfahrungen, aber auch, dass das im Alltag enthaltene Widerstandspotenzial gegen restriktive Bedingungen und Ausschlusspraxen zur Sprache kommen kann. Mit dem Interaktionsraum „Filmdiskussion" beabsichtigten wir, einen möglichst selbstgesteuerten und geschützten Dialog auch - aber nicht nur - zur Thematisierung ,heikler“ bzw. ,sozial unerwünschter" Aspekte anzubieten.

Durch die Verknüpfung qualitativer Forschungsmethoden Sozialer Arbeit, sozialwissenschaftlich fundierter Rekonstruktiver Sozialpädagogik (Jakob und Wensierski 1997), verstehender und fallrekonstruktiver Sozialer Arbeit (Loch und Schulze 2010; Völter 2008) und einer hermeneutisch-reflexiven Methodenreflexion (Groddeck und Schumann 1994) wurde ein Zugang zu den Alltagserfahrungen geflüchteter Jugendlicher gesucht. Statt zu kolonisieren (Holzkamp 1995) oder expertokratisch vorab entwickelte Relevanzen der Forscherinnen ,abzufragen“, ging es uns im Anspruch emanzipatorischer Praxisforschung (Markard 2018) und Bildungsarbeit darum, subjektive Probleme mit gesellschaftlichen Lebensverhältnissen in Verbindung zu brin- 
gen. Auf diesem Weg wurde die Haltung des forschenden Nachvollziehens der inneren Logiken von Handlungen, Deutungen, Interaktions- und Lebenskontexten der Jugendlichen eingenommen, ,um die Vereinnahmung fremder Lebenswelten und andere Machtwirkungen des ,Verstehens“ zu vermeiden“ (Völter 2008, S. 2). Damit wird versucht, das Theorie-Praxis-Verhältnis im Sinne eines intermediären Feldes eines Wissenschaft-Praxis-Diskurses zu präsentieren und qualitative Forschung für die Praxis Sozialer Arbeit situationsanalytisch fallorientiert entgegen nomothetischer Verallgemeinerungen fruchtbar zu machen. Des Weiteren beabsichtigten wir, diese für Bildungsprozesse im Rahmen des Studiums Sozialer Arbeit zur Ausbildung einer ,ethnografische[n] Kompetenz und Bildung“ (Völter 2008, S. 1) zu nutzen, und haben daher mit einer internationalen studentischen Auswertungsgruppe zusammengearbeitet (s. oben).

Die Auswertung ergab, dass die Jugendlichen sich innerhalb dieser Filmdiskussion als Gruppe wahrnahmen. Der Film ermöglichte ihnen damit eine Kollektiverfahrung, u. a. in Bezug auf Generation, Gender und Herkunft: „Ja, die Frauen haben auch gezeigt, dass die Frauen nix alleine sind, und die haben gezeigt, dass Männer und Frauen sind zusammen. Wenn sie zusammenarbeiten, dann machen sie alles. Dann schaffen sie alles, was sie arbeiten wollen“ (Jugendlicher 4, Z. 194-197).

Deutlich wurde in der Analyse, dass die Diskussionsteilnehmer*innen in unserer Filmdiskussion an verschiedenen Stellen offenbar eine Außenperspektive einnahmen, denn sie äußerten abstrakte Analysen des Phänomens und des subjektiven Umgangs mit Diskriminierung: „Ja, aber zum Beispiel jeden Gruppe gibt es viele talente Mann oder talente Frau oder gibt es normale Menschen. Zum Beispiel sie ist ganz normale im Leben. Aber jetzt wir haben gesehen im Film drei schwarze Frauen, sie waren so talent (gemeint ist talentiert, HS/TG), und sie konnten etwas Gutes machen, sehr guter als andere, aber das ist nicht alles. Weil viele andere Menschen gibt es, was sehr normal ist. Okay, wir klatschen für talente Frau, aber andere auch schwarz. Aber sie sind schwarz, egal, sie sind so talent, wir brauchen sie. Aber andere sind egal. Auch schwarz. Wir können nicht zusammen reden oder zusammen trinken. Wir brauchen nur die. Ich glaube auch nicht so fertig die Rassismusprobleme“ (Jugendlicher 2, Z. 628-637). Der Jugendliche spricht hier nicht nur über die Protagonist*innen des Films, sondern er tritt ein für das Grundrecht auf Anerkennung jedes Menschen ungeachtet besonderer Fähigkeiten, als Gegenentwurf zum Film, in der „Schwarze ${ }^{2}$ Menschen“ sich über besondere Leistungen unter Beweis stellen müssen um anerkannt zu werden und thematisiert damit die kompensatorischen Zumutungen an geflüchtete Menschen in Anbetracht der Etabliertenvorrechte und der sozialer Ungleichheit aufgrund des markierten Nicht-Weißseins.

In Bezug auf die Erzählungen über Alltagserfahrungen jedoch zeigt sich, dass die Jugendlichen eigene Ausgrenzungserfahrungen häufig auf der kognitiven Ebene relativieren bzw. legitimieren. „Das ist Schuld von Ausländer“, hatte ein Jugendlicher gesagt (s. oben), ,wir müssen was gute machen für die Deutschland“ (Jugendlicher 4, Z. 647-649).

\footnotetext{
2 Der Begriff Schwarz wird hier extra großgeschrieben, um diesen von der Essentialität einer Hautfarbe abzugrenzen, sondern soll vielmehr die soziale Konstruktion einer sozialpolitischen Positionierung, die daraus hergestellt wird verdeutlichen.
} 
Die Auswertung der Daten zeigte bei den teilnehmenden jungen Geflüchteten eine deutliche Diskrepanz zwischen generalisierenden Einschätzungen zu Diskriminierung einerseits und den konkreten, alltäglichen und persönlichen Rassismuserfahrungen andererseits, z.B.: (1) „Rassismus findet statt, aber nicht in Europa, da hier Regeln existieren“ - die Alltagserfahrung, z. B. bei der Wohnungssuche, hingegen lässt die Jugendlichen zu dem Schluss kommen, dass es in Europa ,ein bisschen Rassismus“ (Jugendliche 2, Z. 296) gibt. (2) „Heute gibt es weniger Rassismus als früher, u.a. wegen inzwischen verbriefter Rechte" - ihre Alltagserfahrungen zeigen ihnen jedoch, dass offizielle Rechte keineswegs automatisch deren konkrete Umsetzung zur Folge haben. (3) „Rassismus hört auf, sobald ein Pass vorliegt“ - ihre Alltagserfahrung führt jedoch eine Reihe ,struktureller“ Benachteiligungen vor Augen, z. B. in Bezug auf den Zugang zu Bildung. In den Worten einer Teilnehmerin: „Und okay, wir sind gegen Rassismus, und wir haben richtig Schule, und wir haben so viel Rechte, aber, in die gleiche Satz, wir haben nicht so was wir denk“ (Jugendliche 2, Z. 339-341).

\subsection{Narrativ orientierte Gesprächsführung der Gruppen-/Filmdiskussion: Eröffnung eines Zugangs zu biografischen Selbstthematisierungen}

Unser Zugang mit dem Medium Film als Impulsgeber für subjektive wie kollektive thematische Diskussions- und Anknüpfungspunkte, aber auch als Anregung für alltags- und lebensweltliche biografische Selbstthematisierungen im Rahmen einer Gruppendiskussion zielte auf eine ethnografische Erkenntnishaltung (vgl. ausführlich Alheit 2001; Schütze 1994). Die Gesprächsführung orientierte sich daher an der Methode des narrativen Interviews und lässt sich durch ein offenes Vorgehen und eine ethnografische Haltung der Moderator*innen charakterisieren.

Anhand der Gesprächsführung sollte ein Artikulationsraum eröffnet und aufrechterhalten werden, der selbststrukturierte Erzählungen ermöglicht, ohne auf weitere Interventionen der Moderation angewiesen zu sein. Die Orientierung erfolgt dabei zunächst an den alltagsweltlichen Konstruktionen der Gesprächspartner*innen. Die narrative Gesprächsführung zielt darauf, den Gesprächsbeteiligten einen größtmöglichen Raum zu selbstgestalteten Erzählungen über eigene Erfahrungen zum angesprochenen Thema zu geben und durch gezeigtes Interesse und parasprachliche Aufmerksamkeitsbekundungen unterstützend zu wirken. Fragen zielen im Sinne einer sozialen Handlungsorientierung eher auf erfahrene singulär-situative Handlungsabläufe als auf statische Beschreibungen und argumentative Begründungen (Loch und Schulze 2010). Wie in jedem narrativen Interview, so stand auch zu Beginn der Gruppendiskussion eine offene Erzählaufforderung - , Was haben Sie denn beim Zuschauen des Films so gedacht?“ -, um die Teilnehmer*innen darin zu unterstützen, sich ihrem Erinnerungs- und Erzählfluss zu überlassen.

Im wissenschaftlichen Prozess bedient sich das narrative Interview der Alltagskompetenz des Erzählens, über das die meisten Menschen verfügen. Erzählen ist eine Ressource des Sich-verständlich-Machens und der Selbstvergewisserung - in einer Forschungssituation wie auch im Alltag. Erzählungen können während des Kommunikationsverlaufs Selbst- und Fremdverstehensprozesse unterstützen sowie manifest und latent wirksame Handlungsorientierung verstehbar machen, und im alltäglichen 
Erzählen können Handlungen und Ereignisse ausgesprochen werden, die ansonsten eher vermieden werden (Loch und Schulze 2010). Für die Gesprächspartner*innen kann das Thematisieren dieser Erfahrungen erleichternd sein. Letzteres waren unsere Ausgangsüberlegungen für unsere Gruppendiskussionen, mit dem Ziel, eine offene und vertrauensvolle Gesprächsatmosphäre zu schaffen und mit offenen Fragen die Teilnehmenden im freien Erzählen zu unterstützen.

Die Aussagen der Jugendlichen sollten vom Standpunkt einer ethnografischen Neugier, einer reflexiven Befremdung in ihrer Besonderheit betrachtet werden (das Besondere im Allgemeinen bzw. das Allgemeine im Besonderen), d.h., sie sollten nicht vorschnell in vertraute (Problem-)Kategorien überführt werden. Durch das Fremdmachen und das Wiederannähern mit einem befremdenden Blick wurde eine ethnografische Erkenntnishaltung während des gesamten Prozesses aufrechterhalten. Mit dieser Haltung, nicht zu wissen, was das Gegenüber meint, werden Erzählende dazu angeregt, ihre eigenen Bedeutungszuschreibungen mit eigenen Worten auszusprechen, mit der Intention, die die Erzählende*n in ihrer Autor*innenschaft über ihr (Er)Leben zu unterstützen (Schulze et al. 2020). Wichtig dabei ist, die Sinnzuschreibungen und Wortbedeutungen mit den Erzählenden gemeinsam im dialogischen Austausch zu verstehen. So wird der Akt des Erzählens wegen seiner Alltäglichkeit nicht etwa trivialisiert, sondern es geht gerade darum, einen Raum hierfür zu eröffnen und alltägliche Äußerungen durch systematisches Fremdmachen neu zu verstehen.

Neben einem grundsätzlichen Interesse an der Perspektive der Teilnehmenden der Gruppen-/Filmdiskussion ist daher im Falle einer narrativen Orientierung von der Moderation gefordert, keine manipulierenden bzw. Suggestivfragen zu stellen und die geäußerten Meinungen nicht zu bewerten. Insbesondere muss vermieden werden, den teilnehmenden geflüchteten Jugendlichen die Definitionsmacht für ihr Erlebtes zu nehmen und einen Assimilationsdruck zu erzeugen (Kupfer 2018) oder die Fragestellungen in einer Wir (Mehrheitsangehörige) - Ihr (Geflüchtete)-Semantik zu formulieren (z.B. durch die Frage ,Wie ist das bei Euch so?“").

Während der Gruppen-/Filmdiskussion beschränkte sich die Moderation auf Ermunterungen der Jugendlichen, sich an der Diskussion zu beteiligen, sowie auf Verständnisfragen und das Aufzeigen von Unterschieden in den Erfahrungen und Einschätzungen der Teilnehmenden. Der narrativ orientierte Moderationsstil überließ alle inhaltlichen Relevanzsetzungen den Jugendlichen selbst. Im Wesentlichen zielte die Moderation auf die Herstellung einer von Anerkennung getragenen Gesprächssituation und unterstützte die Thematisierung eigener Erfahrungen, Meinungen und Einschätzungen, auch in Fällen, wenn dies von der Gruppe nicht geteilt wurde.

Es zeigte sich, dass insbesondere diese Haltung die Thematisierung von Rassismus- bzw. Diskriminierungserfahrungen ermöglichte: Nachdem z. B. eine Teilnehmerin klar gesagt hatte, in Europa gebe es ihrer Ansicht nach keinen Rassismus mehr (Jugendliche 4, Z. 288), wandte sich die Moderatorin einladend an die gesamte Gruppe mit den Worten „Wie ist das? Sehen Sie das auch so? Also, sprechen Sie ruhig miteinander. Einfach, wer 'ne Idee hat und was sagen möchte“ (Z. 293-294), woraufhin ein anderes Gruppenmitglied eine kritische Aussage wagte: „Ich bin nicht total die gleiche Mein, aber er hat ein bisschen stimmt. Aber auch heute hier in Eu- 
ropa gibt es noch ein bisschen Rassismus. An so viel Dinge kann man das sehn, oder?" (Jugendliche 2, Z. 295-297).

\section{Zusammenfassung und Ausblick}

Deutlich wurde in der Filmdiskussion des geschilderten Projekts, dass die gemeinsame Erfahrung des Filmschauens und die sich anschließende offene, anerkennungssowie narrativ orientierte Gruppendiskussion den geflüchteten jungen Menschen eine Beobachter*innenposition eröffnete und damit eine analytische Perspektive auf die Situation der Filmfiguren, aber ebenso auf Ähnlichkeiten zum eigenen Leben. Damit wurde bei ihnen auch eine kritische Reflexion eigener Erfahrungen auf individueller wie struktureller Ebene angeregt, insbesondere zum Phänomen der Diskriminierung. Die Gruppendiskussion ermöglichte den einzelnen Teilnehmenden die Erkenntnis, dass die eigenen Erfahrungen von anderen geteilt werden.

Dies initiiert in der Folge einen diskursiv angelegten Bildungsprozess im Sinne des transformatorischen Ansatzes, der Bildung in der gesamten Lebensgeschichte der Person verortet und in „kognitiven Figuren“ oder „Deutungsmustern“ grundlegende Figuren des Welt- und Selbstverhältnisses berührt sieht (vgl. Sting 2018, S. 408). Ein Ziel dieses Bildungsprozesses besteht darin, zu kritischer Reflektiertheit und Selbstbestimmung beizutragen (vgl. Fuchs 2014). Ein solches Bildungsverständnis ist daher auch als Streben nach sozialer Inklusion und als Gestaltung des sozialen Zusammenlebens zu verstehen. Diese Sichtweise wird vom Ansatz H.-R. Müllers (2014) unterstützt, demzufolge Bildung neben Selbstentfaltung auch ,Vergemeinschaftungspraktiken“ (Müller 2014, S. 98) und soziale Zugehörigkeit und Anerkennung anstrebt. „Die Theorie transformatorischer Bildungsprozesse eröffnet damit - je nach Perspektive - die Möglichkeit, nicht nur soziale Voraussetzungen von Bildungsprozessen und deren Abhängigkeit von Anerkennungsprozessen zu reflektieren, sondern auch die soziale Zielstellung von Bildung als Streben nach sozialer Inklusion und Gestaltung des sozialen Zusammenlebens zu thematisieren“ (Sting 2018, S. 408; vgl. auch Walter 2014; Zeller 2012). Damit zeigt sich die von uns vorgestellte Methode einer narrativ-orientierten „Filmdiskussion“ als an Paulo Freires (1973) Konzept der „Pädagogik der Unterdrückten“ orientiert. Ziel ist die individuelle Bewusstmachung und Veränderung ausgrenzender Strukturen auf Basis einer dialogischen, problemformulierenden Bildungsarbeit. Entscheidend hierzu sei, so P. Freire (1973), die kontextuelle Dimension der Realität herauszuarbeiten und in ihren Interaktionen zu begreifen. Wird dies mit der Methodologie der Bewusstmachung ausgeführt, so leite dies die Menschen zum kritischen Denken über die Welt an (Freire 1973, S. 86-88).

Ziel unseres Projekts war, einen subjektorientierten dialogischen, insbesondere nicht paternalistischen (Scherr 2002) Raum durch einen die Jugendlichen ansprechenden Film zu geben. Eine solche film- und problemfokussierte Bildungsarbeit folgt dem Anspruch, ein Bewusstsein darüber zu erzeugen, dass die subjektiven Erfahrungen, das Denken darüber und auch das darauf bezogene Handeln oder Nichthandeln durch gesellschaftliche Verhältnisse hervorgebracht werden. Wichtig hierbei ist, nicht mit einem (Besser-)Wissen von außen an die Teilnehmenden her- 
anzutreten, sondern das gemeinsame „Leiden an den Verhältnissen“ erfahrbar zu machen, um eine gesellschaftliche Ebene über Lebens-, Differenz- und Machtverhältnisse im Ankunftsland zu erkennen und einzugreifen. Denn, so U. Osterkamp (2018) in ihrer Reflexion eines subjektwissenschaftlichen Praxisforschungsprojekts mit Geflüchteten: „Wenn du aber diese subjektive Ebene weglässt, wird das Politische zu einer abstrakten Anforderung, der man sich, wie allen fremdbestimmten Anforderungen, so weit wie möglich zu entziehen sucht“ (Osterkamp 2018, S. 94). Die Herausforderung für die Praxis besteht darin, die subjektive Zuwendung zu den konkreten Lebensbedingungen zu fördern, um emanzipatorische Bildungsprozesse zu fördern. So müsste die Aussage eines Teilnehmers in der Gruppendiskussion: „Jedermann kann [...] etwas so machen [...] - egal [...] er hat Hautfarbe [...] schwarze Farbe oder weiße Farbe oder rote Farbe" (Jugendlicher 3, Z. 136-137) durch narratives Nachfragen an die Konkretheit der eigenen Lebenserfahrung rückgebunden und Sprachraum über lebensgeschichtliche Differenzerfahrungen entgegen affirmativ-habitueller Abstraktionen reflexiv gestaltet werden.

Mit unserem Artikel zeigen wir das Potenzial der Verknüpfung rekonstruktiver Forschungsmethoden mit der Praxismethode Filmdiskussion in der Sozialen Arbeit mit jugendlichen Geflüchteten auf. Kritisch ist an dieser Stelle anzumerken, dass wir im Rahmen dieses explorativen Projektes die Heterogenität der Gruppe der ,,jugendlichen Geflüchteten“ vernachlässigt haben. Anschließende Analysen müssten stärker Differenzierungen - u. a. im Hinblick auf soziale Positionierungen aufgrund der Geschlechter* - in den Blick nehmen. Die aktuelle Fokussierung auf die Themen Flucht- und Diskriminierungserfahrungen und deren Bedingungen leitet sich neben dem Stimulus - nicht zuletzt aus unseren Forschungsinteressen ab. Wir sind uns dessen gewahr, dass wir den Film aus der Perspektive einer diskriminierungskritischen Sozialen Arbeit ausgewählt haben und die geflüchteten Jugendlichen damit zugleich in Diskriminierungsstrukturen positioniert haben. Für uns ist dies eine unvermeidbare Paradoxie zwischen emanzipatorischer Bewusstmachung (Freire 1973) und eines nach sich ziehenden Othering-Prozesses, der immer mit der Zuwendung zu einer Zielgruppe verbunden ist. Aus dieser Reflexion leiten wir ab, die Filmauswahl zukünftig den Jugendlichen zu überlassen, deren Selbstbestimmung ernst zu nehmen und offen für die Wahl ihrer biografisch situierten Themen zu sein. Dies auch, um nicht einem eurozentristischen Adoleszenzkonzept (wie im Fall unserer Coming-of-Age-Filme) zu verfallen und damit vermeintliche Normalitätsvorstellungen auf zu oktroyieren, zumal (auch) geflüchtete Jugendliche internationale Filme über Streaming-Dienste rezipieren.

Die Praxisforschung ermöglichte uns eine datengestützte Reflexion - im Wissen, dass die Durchführung immer schon eine Intervention durch Interaktion (Moderation zu Gruppe sowie Gruppeninteraktion) und nicht nur eine abstinente Form der Datenerhebung darstellt. Die Aussagen der Jugendlichen sind in Koproduktion mit den anderen Teilnehmer*innen und den Moderatorinnen entstanden und müssen als situative Wissensbildung verstanden werden. Es leiten sich hieraus keine universellen Wahrheiten über geflüchtete junge Menschen ab. Die reflexive Herstellung und Aufrechterhaltung nicht kolonisierender Artikulationsräume sollte u.E. daher verstärkt und kontinuierlich Aufgabe emanzipatorischer und transformativer Bildung im Kontext von Migration, Flucht und Sozialer Arbeit sein, um Menschen im Hin- 
blick auf deren Überwindung restriktiver Bedingungen zu ihrer Selbstbestimmung und Erweiterung ihrer Handlungsfähigkeit zu unterstützen.

Funding Open Access funding provided by Projekt DEAL.

Open Access Dieser Artikel wird unter der Creative Commons Namensnennung 4.0 International Lizenz veröffentlicht, welche die Nutzung, Vervielfältigung, Bearbeitung, Verbreitung und Wiedergabe in jeglichem Medium und Format erlaubt, sofern Sie den/die ursprünglichen Autor(en) und die Quelle ordnungsgemäß nennen, einen Link zur Creative Commons Lizenz beifügen und angeben, ob Änderungen vorgenommen wurden.

Die in diesem Artikel enthaltenen Bilder und sonstiges Drittmaterial unterliegen ebenfalls der genannten Creative Commons Lizenz, sofern sich aus der Abbildungslegende nichts anderes ergibt. Sofern das betreffende Material nicht unter der genannten Creative Commons Lizenz steht und die betreffende Handlung nicht nach gesetzlichen Vorschriften erlaubt ist, ist für die oben aufgeführten Weiterverwendungen des Materials die Einwilligung des jeweiligen Rechteinhabers einzuholen.

Weitere Details zur Lizenz entnehmen Sie bitte der Lizenzinformation auf http://creativecommons.org/ licenses/by/4.0/deed.de.

\section{Literatur}

Alheit, P. (2001). „Ethnografische Pädagogik“. Eine andere Sichtweise des pädagogischen Feldes. Die Deutsche Schule, 93(1), 10-16.

Barthelmes, J., \& Sander, E. (2001). Erst die Freunde, dann die Medien. Medien als Begleiter in Pubertät und Adoleszenz. Reihe: Medienerfahrungen von Jugendlichen, Bd. 2. München: DJI.

Bierman, J.S., Krieger, A.R., \& Leifer, M. (2003). Group cinematherapy as a treatment modality for adolescent girls. Residential Treatment for Children \& Youth, 21(1), 1-15.

Bilden, H. (2007). Neue Medien - Sozialisation - Geschlecht. Markt Indersdorf. http://www.helga-bilden. de/Artikel/Neue_Medien-Sozialisation-Geschlecht.html. Zugegriffen: 15. Febr. 2019.

Bohnsack, R. (2007). Rekonstruktive Sozialforschung. Einführung in qualitative Methoden (6. Aufl.). Opladen: utb.

Bonfadelli, H. (1983). Der Einfluss des Fernsehens auf die Konstruktion der sozialen Realität: Befunde aus der Schweiz zur Kultivierungshypothese. Rundfunk und Fernsehen, 31(3-4), 415-430.

Bourdieu, P. (1990). Was heißt sprechen? Zur Ökonomie des sprachlichen Tausches. Wien: Braumüller.

Bundesjugendkuratorium (BJK) (2009). Partizipation von Kindern und Jugendlichen - Zwischen Anspruch und Wirklichkeit. München. http://www.bundesjugendkuratorium.de/assets/pdf/press/bjk_ 2009_2_stellungnahme_partizipation.pdf. Zugegriffen: 15. Febr. 2019.

Bundeszentrale für politische Bildung (bpb) (2016). Filmarbeit mit geflüchteten und einheimischen Kindern und Jugendlichen. https://www.kinofenster.de/download/2016-dossier-filmarbeit.pdf. Zugegriffen: 15. Febr. 2019.

Butler, J. (2001). Psyche der Macht. Das Subjekt der Unterwerfung. Frankfurt a.M.: Suhrkamp.

Butler, J. (2006). Haß spricht. Zur Politik des Performativen. Frankfurt a.M.: Suhrkamp.

Dörr, M. (2008). Erwachsene. In A. Hanses \& H. G. Homfeldt (Hrsg.), Lebensalter und Soziale Arbeit. Eine Einführung (S. 174-191). Baltmannsweiler: Schneider Hohengehren.

Elias, N., \& Scotson, J.L. (2006). Etablierte und Außenseiter (Bd. 4). Frankfurt a.M.: Suhrkamp.

Festinger, L. (1954). A theory of social comparison processes. Human Relations, 7(2), 117-140.

Flick, U. (2009). Qualitative Sozialforschung. Eine Einführung (Bd. 2). Reinbek bei Hamburg: Rowohlt.

Fraser, N. (1994). Widerspenstige Praktiken. Macht, Diskurs, Geschlecht. Frankfurt a.M.: Suhrkamp.

Freire, P. (1973). Pädagogik der Unterdrückten. Bildung als Praxis der Freiheit. Reinbek bei Hamburg: Rowohlt.

Fuchs, M. (2014). „Das war das bedeutendste daran, dass ich mich so verändert habe.“ Mit Ehrgeiz und Ansporn über Umwege zum Ziel - der „Bildungsweg“ Hakans. Oder: Ist jede Transformation von Welt- und Selbstverhältnissen sogleich bildungsbedeutsam? In H.-C. Koller \& G. Wulftange (Hrsg.), Lebensgeschichte als Bildungsprozess? Perspektiven bildungstheoretischer Biographieforschung (S. 127-151). Bielefeld: transcript. 
Gerbner, G., \& Gross, L. (1976). Living with television: The violence profil. Journal of Communication, 26(2), 173-199.

Grendel, T. (2018). Die Kategorie „Flüchtling“ als Begrenzung gesellschaftlicher Teilhabe? Erfahrungen gesellschaftlicher Zuschreibungen aus der Perspektive jugendlicher Geflüchteter. пеие praxis, 48(3), 201-220.

Groddeck, N., \& Schumann, M. (1994). Modernisierung Sozialer Arbeit durch Methodenentwicklung und Methodenreflexion. Freiburg: Lambertus.

Hébert, T.P., \& Neumeister, K.L.S. (2001). Guided viewing of film: A strategy for counseling gifted teenagers. Journal of Secondary Gifted Education, 12(4), 224-235.

Hébert, T. P., \& Neumeister, K. L. S. (2002). Förderung der sozialen und emotionalen Entwicklung begabter Kinder durch eine geführte Filmbetrachtung. Roer Off, 25(1), 17-21.

Heitmeyer, W. (2002). Gruppenbezogene Menschenfeindlichkeit. Die theoretische Konzeption und erste empirische Ergebnisse. In W. Heitmeyer (Hrsg.), Deutsche Zustände. Folge 1 (S. 15-31). Berlin: Suhrkamp.

Holzkamp, K. (1985). Grundlegung der Psychologie. Studienausgabe. Frankfurt a.M.: Suhrkamp.

Holzkamp, K. (1995). Kolonisierung der Kindheit. Psychologische und psychoanalytische Entwicklungserklärungen. Forum Kritische Psychologie, 18(1), 109-131.

Horvath, K. (2017). Die Illusion der Andersartigkeit: mit Bourdieu zu einer rassismuskritischen Bildungsforschung? In M. Rieger-Ladich \& C. Grabau (Hrsg.), Pierre Bourdieu: Pädagogische Lektüren (S. 147-166). Wiesbaden: Springer VS.

IOM - International Organization for Migration (2013). Children on the move. http://publications.iom.int/ bookstore/free/Children_on_the_Move_15May.pdf. Zugegriffen: 15. Febr. 2019.

Jakob, G., \& von Wensierski, H.-J. (1997). Rekonstruktive Sozialpädagogik. Konzepte und Methoden sozialpädagogischen Verstehens in Forschung und Praxis. Weinheim: Beltz Juventa.

Kämpf, H. (2006). Judith Butler: Die störende Wiederkehr des kulturell Verdrängten. In S. Moebius \& D. Quadflieg (Hrsg.), Kultur. Theorien der Gegenwart (S. 246-256). Wiesbaden: VS.

Kilomba, G. (2009). Das N-Wort. In Bundeszentrale für politische Bildung (Hrsg.), Afrikanische Diaspora in Deutschland. Bonn: bpb.

Kupfer, A. (2018). Rassismus in Beratung. In H. Schulze, D. Höblich \& M. Mayer (Hrsg.), Macht Diversität - Ethik in der Beratung. Wie Beratung Gesellschaft macht (S. 74-93). Opladen: Barbara Budrich.

Lamnek, S. (2005). Gruppendiskussion. Theorie und Praxis (2. Aufl.). Weinheim: Juventa.

Langton, R. (2007). Sprechakte und unsprechbare Akte. In S. K. Herrmann, S. Krämer \& H. Kuch (Hrsg.), Verletzende Worte. Die Grammatik sprachlicher Missachtung (S. 107-146). Bielefeld: transcript.

Liebel, M. (2015). Kinderinteressen. Zwischen Paternalismus und Partizipation. Weinheim: Beltz Juventa.

Litau, J., Walther, A., Warth, A., \& Wey, S. (Hrsg.). (2016). Theorie und Forschung zur Lebensbewältigung. Weinheim: Beltz Juventa.

Loch, U., \& Schulze, H. (2010). Biografische Fallrekonstruktion im handlungstheoretischen Kontext der Sozialen Arbeit. In W. Thole (Hrsg.), Grundriss Soziale Arbeit. Ein einführendes Handbuch (3. Aufl. S. 687-706). Wiesbaden: VS.

Luca, R. (Hrsg.). (2003). Medien.Sozialisation.Geschlecht. Fallstudien aus der sozialwissenschaftlichen Forschungspraxis. München: kopaed.

Luca, R., \& Decke-Cornill, H. (2010). Jugend - Film - Gender. Medienpädagogische, bildungstheoretische und didaktische Perspektiven. Stuttgart: ibidem.

Lucius-Hoene, G., \& Deppermann, A. (2002). Rekonstruktion narrativer Identität. Ein Arbeitsbuch zur Analyse narrativer Interviews. Opladen: Barbara Budrich.

Markard, M. (2018). Das Theorie-Praxis-Problem in (der Geschichte) der Kritischen Psychologie. Forum Kritische Psychologie, 41(1), 7-16.

Mecheril, P. (2010). Migrationspädagogik. Hinleitung zu einer Perspektive. In P. Mecheril, M. M. Castro Varela, I. Dirim, A. Kalpaka \& C. Melter (Hrsg.), Migrationspädagogik (S. 7-22). Wiesbaden: VS.

Melter, C. (2006). Rassismuserfahrungen in der Jugendhilfe. Eine empirische Studie zu Kommunikationspraxen in der Sozialen Arbeit. Münster: Waxmann.

Müller, A.-L. (2011). Worte schaffen Soziales: Wie Sprache Gesellschaft verändert. http://www.journalfuer-psychologie.de/index.php/jfp/article/download/14/77. Zugegriffen: 15. Febr. 2019.

Müller, H.-R. (2014). „Aufstieg durch Bildung“ - Versionen der Selbstbeschreibung im Interview mit Hakan Salman. In H.-C. Koller \& Wolftange (Hrsg.), Lebensgeschichte als Bildungsprozess? Perspektiven bildungstheoretischer Biographieforschung (S. 79-101). Bielefeld: transcript.

Neckel, S. (1991). Status und Scham. Zur Symbolischen Reproduktion sozialer Ungleichheit. Frankfurt a.M.: Suhrkamp. 
Niedrig, H., \& Seukwa, H. (2010). Die Ordnung des Diskurses in der Flüchtlingskonstruktion. Eine postkoloniale Re-Lektüre. Diskurs Kindheits- und Jugendforschung, 5(2), 181-193.

Osterkamp, U. (2018). „Rassismus als Selbstentmächtigung“? - Einblicke in die Arbeit des Projektes Rassismus \& Diskriminierung. Interview von Santiago Vollmer und Thomas Pappritz. Forum Kritische Psychologie, 41(1), 83-97.

Rappaport, J. (1995). Empowerment meets narrative: Listening to stories and creating stories. American Journal of Community Psychology, 23(5), 795-808.

Rommelspacher, B. (1995). Dominanzkultur. Texte zu Fremdheit und Macht. Berlin: Campus.

Rommelspacher, B. (2006in). Wie wirkt Diskriminierung? Vortrag bei der Tagung des Instituts Mensch, Ethik und Wissenschaft ,Ethik und Behinderung - Vom Paradigmenwechsel zur Praxis der Anerkennung“, am 12.05.2006 in Berlin. http://www.imew.de/index.php?id=319. Zugegriffen: 15. Febr. 2019.

Rosenthal, G. (2002). Grundzüge der Ethnographie und die Grenzen verbaler Daten. In G. Müller-Mundt \& D. Schaeffer (Hrsg.), Qualitative Gesundheits- und Pflegeforschung (S. 133-147). Bern: Huber.

Scherr, A. (2002). Abschied vom Paternalismus. Anforderungen an die Migrationssozialarbeit in der Einwanderungsgesellschaft. In A. Treichler (Hrsg.), Wohlfahrtsstaat, Einwanderung und ethnische Minderheiten (S. 187-198). Wiesbaden: VS.

Schramkowski, B. (2007). Für mich aber hat dieses Integrationswort mit der Zeit seinen Wert verloren - Perspektiven junger Erwachsener mit Migrationshintergrund. In T. Geisen \& C. Riegel (Hrsg.), Jugend, Partizipation und Migration (S. 149-167). Wiesbaden: VS.

Schramkowski, B., \& Ihring, I. (2018). Alltagsrassismus. (K)ein Thema für die Soziale Arbeit? In B. Blank, S. Gögercin, K. Sauer \& B. Schramkowski (Hrsg.), Soziale Arbeit in der Migrationsgesellschaft (S. 279-290). Wiesbaden: Springer VS.

Schulze, H. (2009). Narration und Figuration. Zum Aspekt biografischer, kultureller und institutioneller Interdependenz beim Erzählen von Krankheitserleben im Kontext von Migrationserfahrung. In G. Grimm (Hrsg.), Erzählen, Träumen und Erinnern. Erträge klinischer Erzählforschung (S. 298-306). Lengerich: Pabst Science Publishers.

Schulze, H., \& Witek, K. (2014). Beschämung statt Bildung? Wie im Beratungsgespräch aus Missachtungserfahrungen Selbstwirksamkeit konstruiert wird. Sozial Extra, 38(3), 50-53.

Schulze, H., Mantey, D., \& Witek, K. (2016). Sprache und Gewalt: Zu Mikropraktiken von Alltagsrassismus in der Beratung. In C. Equit, A. Groenemeyer \& H. Schmidt (Hrsg.), Situationen der Gewalt (S. 193-209). Wiesbaden: Springer VS.

Schulze, H., Kühn, M. \& Bialek, J. (2020). Trauma und Lebensbewältigung. Zur Re-Vergewisserung des Sozialen gegen eine Kolonisierung und für das Recht auf Autor*innenschaft der eigenen Geschichte. In G. Stecklina \& J. Wienforth (Hrsg.), Handbuch Soziale Arbeit und Lebensbewältigung. Praxis, Theorie und Empirie. Reihe: Übergangs- und Bewältigungsforschung (S. 585-594). Weinheim: Beltz Juventa.

Schütze, F. (1994). Ethnographie und sozialwissenschaftliche Methoden der Feldforschung. Eine mögliche methodische Orientierung in der Ausbildung und Praxis der Sozialen Arbeit? In N. Groddeck \& M. Schumann (Hrsg.), Modernisierung Sozialer Arbeit durch Methodenentwickung und -reflexion (S. 189-297). Freiburg im Breisgau: Lambertus.

Sting, S. (2018). Bildung. In G. Graßhoff, A. Renker \& W. Schröer (Hrsg.), Soziale Arbeit. Eine elementare Einführung (S. 399-412). Wiesbaden: Springer VS.

Süss, D. (2006). Mediensozialisation zwischen gesellschaftlicher Entwicklung und Identitätskonstruktion. In K.-S. Rehberg (Hrsg.), Soziale Ungleichheit, kulturelle Unterschiede. Verhandlungen des 32. Kongresses der Deutschen Gesellschaft für Soziologie in München (Bd. 2, S. 3370-3380).

Süss, D., Lampert, C., \& Wijnen, C.W. (2013). Medienpädagogik. Ein Studienbuch zur Einfuihrung (2. Aufl.). Wiesbaden: VS.

Thiersch, H. (1978). Die hermeneutisch-pragmatische Tradition der Erziehungswissenschaft. In H. Thiersch, H. Ruprecht \& U. Herrmann (Hrsg.), Die Entwicklung der Erziehungswissenschaft (S. 11-108). München: Juventa.

Thiersch, H. (1992). Lebensweltorientierte soziale Arbeit. Aufgaben der Praxis im sozialen Wandel. Weinheim: Beltz.

United Nations (UN) (1989). Convention on the rights of the child. http://www.ohchr.org/en/ professionalinterest/pages/crc.aspx. Zugegriffen: 15. Febr. 2019.

Völter, B. (2008). Verstehende Soziale Arbeit. Zum Nutzen qualitativer Methoden für professionelle Praxis, Reflexion und Forschung. Forum Qualitative Sozialforschung. https://doi.org/http://dx.doi.org/ 10.17169/fqs-9.1.327. 
Walber, H. (2011). Film-Bildung im Zeichen des Fremden. Ein bildungstheoretischer Beitrag zur Filmpädagogik. Münster: Verlag Westfälisches Dampfboot.

Walter, O. (2014). Immigrant optimism in Deutschland? Die Überprüfung einer Hypothese anhand der mathematikbezogenen Motivation und realistischen Bildungsaspiration von Schülerinnen und Schülern mit Migrationshintergrund. Empirische Pädagogik, 28(1), 36-53.

Weiß, A. (2001). Klasse und Klassifikation. Die symbolische Dimension sozialer Ungleichheit. Wiesbaden: VS.

Weiß, A. (2013). Rassismus wider Willen. Ein anderer Blick auf eine Struktur sozialer Ungleichheit. Wiesbaden: VS.

Zeller, M. (2012). Bildungsprozesse von Mädchen in den Erziehungshilfen. Weinheim: Beltz Juventa.

Ziegler, H. (2015). Soziale Arbeit als Sozialisationsinstanz. In K. Hurrelmann, U. Bauer \& M. Grundmann (Hrsg.), Handbuch Sozialisationsforschung (8. Aufl. ) (S. 453-468). Weinheim: Beltz Juventa. 